\title{
Recomendación del CAVEI sobre la introducción de vacuna contra varicela al Programa Nacional de Inmunizaciones
}

\section{CAVEI recommendation for the introduction of varicella vaccine into the National Immunization Programme}

Jeannette Dabanch'1,2,3, Magdalena Bastías', Cecilia González"1,4, Mario Calvo ${ }^{1,5}$, Johanna Acevedo ${ }^{1,6}$, Jaime Cerda $^{1,7}$, Jaime Rodríguez ${ }^{1,8}$, María Luz Endeiza 1,9, Jaime Inostroza ${ }^{1,10}$, Solange Santillana1,11, Adiela Saldaña ${ }^{1,12}$ y Eduardo Díaz',13

\author{
${ }^{1}$ Comité Asesor de Vacunas y Estrategias de Inmunización, Ministerio de Salud de Chile. \\ ${ }^{2}$ Hospital Clínico Universidad de Chile. \\ ${ }^{3}$ Programa de Enfermedades Infecciosas, Universidad de Valparaíso. \\ ${ }^{4}$ Departamento de Inmunizaciones, Ministerio de Salud de Chile. \\ Instituto de Medicina, Facultad de Medicina, Universidad Austral de Chile. \\ ${ }^{6}$ Departamento de Epidemiología, Ministerio de Salud de Chile. \\ ${ }^{7}$ Departamento de Salud Pública, Facultad de Medicina, Pontificia Universidad Católica de Chile. \\ ${ }^{8}$ Departamento de Infectología, Clínica Alemana de Santiago. \\ ${ }^{9}$ Clínica Universidad de Los Andes. \\ ${ }^{10}$ Facultad de Medicina, Universidad de La Frontera. \\ ${ }^{11 S E R E M I ~ d e ~ S a l u d ~ R e g i o ́ n ~ d e ~ V a l p a r a i ́ s o . ~}$ \\ ${ }^{12}$ Instituto de Salud Pública de Chile. \\ ${ }^{13}$ Facultad de Medicina, Universidad de Chile.
}

$\mathrm{E}$ 1 virus varicela-zoster (VVZ) pertenece a la Familia Herpesviridae y es el agente causal, tanto de la varicela (infección primaria), usualmente en la infancia, como de herpes zoster (reactivación de la infección), generalmente en la vida adulta. Individuos inmunocomprometidos, mujeres embarazadas, recién nacidos y adultos mayores pueden presentar formas graves de varicela o herpes zoster. El VVZ se transmite muy eficientemente de persona a persona por inhalación de gotitas aerosolizadas de secreción respiratoria o fluido de lesiones en piel o por contacto con una lesión de varicela o herpes zoster. Luego de la infección primaria, el virus permanece latente en los ganglios sensoriales y puede reactivarse como herpes zoster. La infección es endémico-epidémica, lo que explica los recurrentes brotes en instituciones cerradas como jardines infantiles, colegios, centros asistenciales, entre otros. Estas infecciones son posibles de prevenir con el uso de vacunas contra el VVZ ${ }^{1,2}$.

Si bien la erradicación del VVZ no es posible con las vacunas disponibles porque se componen de virus vivo atenuado, son neurotrópicas, con posibilidad de latencia y reactivación viral causante del herpes zoster, la vacunación contra varicela en grupos de riesgo o en forma universal reduce la incidencia y hospitalizaciones por enfermedades causadas por VVZ. En el caso de la vacunación universal, ésta reduce la frecuencia de enfermedades por VVZ, tanto en individuos vacunados como en aquellos no vacunados. Algunos sujetos vacunados podrían desarrollar la enfermedad, especialmente aquellos que recibieron sólo una dosis, pero, en estos casos, la manifestación del cuadro es leve o atenuada (menos de 50 lesiones y posiblemente afebril) ${ }^{1,3-6}$.

La Organización Mundial de la Salud (OMS) promueve la vacunación universal contra varicela cuando la infección por VVZ se considera un problema de salud pública o si se justifica por su impacto socioeconómico ${ }^{1}$.

En consideración de los siguientes antecedentes: 


\section{Epidemiología de varicela en Chile}

La vigilancia centinela de varicela comenzó el año 2006, basada en el diagnóstico clínico de la enfermedad en 21 establecimientos de atención primaria de todas las regiones de Chile. Su representatividad es de 5\% del nivel nacional.

- Entre los años 2007 y 2018, la incidencia de varicela ha sido oscilante, con un mínimo de 16/10.000 habitantes el año 2008 y un máximo de 39,4/10.000 en el 2011. El año 2019 la incidencia fue de 19,7/10.000. Según grupo de edad, los casos de varicela se concentran en los niños entre 1 y 9 años. Según región del país, la incidencia de varicela es heterogénea, diferencias que varían según año.

- Durante el año 2019, se notificaron 1.839 casos de varicela en los 21 centros centinela del país, con una tasa de notificación de 19,7 casos por 10.000 habitantes.

- El año 2019 se notificaron 61 brotes de varicela, 72\% de ellos ocurrió en instituciones como jardines infantiles y colegios. A la semana epidemiológica número 5 del año 2020, no se han reportado brotes.

- Entre los años 2006 y 2016, las defunciones anuales por varicela variaron de un mínimo de 3 muertes el 2012 a un máximo de 9 el 2011.

- Los egresos hospitalarios con diagnóstico de varicela presentaron una tendencia a la disminución entre el 2006 y el 2017, desde aproximadamente 1.200 casos a alrededor de 600 , y se concentraron en el niño de 0 a 4 años.

\section{Carga económica directa e indirecta de la varicela}

En Chile, un estudio retrospectivo de hospitalizaciones por varicela en niños de 0 a 15 años entre enero de 2011 y diciembre de 2015 en cinco hospitales de referencia de la Región Metropolitana y de Valparaíso, identificó 685 casos, equivalentes a 3.065 días de hospitalización acumulados, con una mediana de 3 días por internación. El 7\% de los sujetos ingresó a unidad de tratamientos intensivos, ocupando 190 días/cama, mediana de 3 días. El 6\% ingresó a cuidados intermedios, ocupando 120 días/cama. Los principales diagnósticos de egreso fueron infección de piel y tejidos blandos (28\%), alteraciones neurológicas (7\%) y shock séptico (3\%). La letalidad fue de $0,4 \%$. Según arancel del sistema público de salud de Chile, las 685 hospitalizaciones significaron un gasto de USD 268.778, con un costo promedio de USD 392 por episodio. Del total, USD 168.516 fueron en días cama de hospitalización básica, USD 43.434 en días cama de unidad de tratamientos intensivos, USD 13.250 en días cama de cuidados intermedios, USD 12.496 en visitas a urgencia, USD 18.958 en exámenes de laboratorio y USD 12.123 en imágenes. Para el sistema privado de salud se estimó un costo total de USD 1.343 .890 en las 685 hospitalizaciones, con una media de USD 1.960 por episodio ${ }^{8}$. En América Latina, los costos de hospitalización por varicela son variables, lo que se explicaría según costo de día de hospitalización y producto interno bruto per cápita de cada país9.

Un estudio sobre egresos hospitalarios pediátricos por complicaciones de varicela en cuatro centros hospitalarios de Santiago, Chile, que analizó retrospectivamente el período enero de 1997 y febrero de $1999(\mathrm{n}=154)$ mostró que la complicación más frecuente de varicela fue la infección bacteriana de piel y tejidos blandos $(63 \%)$, seguido de infecciones bacterianas invasoras (25\%), complicaciones neurológicas - meningoencefalitis, cerebelitis, convulsión- $(7 \%)$, y de otras complicaciones como herpes zoster, neumonía viral, síndrome purpúrico y artritis reactiva $(4,5 \%)$. El tiempo de hospitalización fue significativamente mayor para las infecciones invasoras $(9,3$ días) en relación con las otras complicaciones (3,5 días infecciones piel y tejidos blandos, 3,6 días neurológicas y 4,1 días misceláneas) $(p=0,001)$. La evolución clínica fue desfavorable en 26 casos: 11 requirieron cirugía, 13 presentaron alguna secuela al momento del alta ( 7 secuela estética, 5 neurológica y 1 ortopédica). Se registraron dos defunciones. La letalidad de la serie completa fue $1,2 \%$ y la de las infecciones invasoras, $5,3 \%{ }^{10}$. El tipo de complicación de varicela y su frecuencia se asemeja a resultados de estudios realizados en Argentina ${ }^{11}$, Perú ${ }^{12}$ y Brasil $1^{13}$, entre otros países de la región.

En cuanto a costos indirectos de varicela por ausentismo escolar y laboral de los padres cuidadores, la descripción de la situación social en torno a la varicela en una comunidad autónoma de España mostró una media de ausentismo escolar de 4,1 días para los niños bajo 15 años de edad y de 0,57 días de ausentismo laboral como consecuencia de la varicela del hijo ${ }^{14}$.

\section{Vacunas contra varicela}

\section{Generalidades}

Las vacunas contra varicela se elaboran en base a virus vivo atenuado, cepa $\mathrm{Oka}^{2,15}$ y se diferencian en el número de unidades formadoras de placas (ufp) -entre $1.000 \mathrm{y}$ 17.000 - y en la cantidad de principio activo por dosis de vacuna. La OMS no especifica el mínimo de ufp por dosis recomendable, pero señala que esto debe ser definido por la entidad regulatoria local.

La vacuna VVZ puede ser monovalente para varicela (V) o combinada para sarampión, rubéola, parotiditis y varicela (SRPV). Todas tienen licencia de uso a partir de los 12 meses de edad, y en el caso de algunos países, una vacuna monovalente y dos vacunas combinadas tienen licencia para uso a partir de los 9 meses. El registro de uso es para la protección contra enfermedad por varicela y no está indicada para proteger contra herpes zoster ${ }^{2}$. 
cualquier grado, que es altamente efectiva (aproximadamente $95 \%$ ) para prevenir enfermedad moderada a grave, y que es altamente efectiva (aproximadamente 99\%) para prevenir enfermedad grave, solamente. La efectividad de dos dosis contra cualquier grado de varicela fue 84 a $98 \%$, con una efectividad media aproximada de 93\%, independiente del tipo de vacuna. En general, dos dosis de cualquier tipo de vacuna varicela entregaron mejor protección contra todo tipo de varicela en comparación a una dosis de cualquier vacuna varicela, 93 y $80 \%$, respectivamente $^{18}$. En Australia, un estudio sobre protección de una dosis de vacuna contra hospitalización por varicela en 10 años de programa mostró una efectividad de $64,7 \%$ $\left(43,3 \% ; 78,0 \% \mathrm{IC}_{95 \%}\right)^{19}$.

Una revisión sistemática sobre las características clínicas y complicaciones de presentaciones de varicela grave en niños vacunados, cuya búsqueda cubrió el período entre los años 1974 y 2016 concluyó que complicaciones graves con diseminación de VVZ y compromiso de otros órganos además de la piel, ocurren pero de forma infrecuente, y que el patrón de complicaciones de varicela post-vacunal es similar al de la varicela en niños no vacunados, siendo las complicaciones más comunes neurológicas, neumonía y sobreinfección bacteriana ${ }^{20}$.

\section{Seguridad}

La vacuna varicela monovalente es bien tolerada, y los efectos adversos reportados con mayor frecuencia en niños sanos incluyen dolor leve, enrojecimiento y erupción cutánea en el sitio de inyección. Comparando esquemas de una y dos dosis, molestias en el sitio de punción se verían aumentadas en la segunda dosis, aunque la fiebre y la erupción cutánea disminuyen ${ }^{2}$.

Una revisión de estudios de seguridad post-licencia de vacuna varicela, realizada por el Comité Consultivo Mundial sobre Seguridad de Vacunas (2012), identificó riesgo de convulsión febril después de la vacunación con SRPV de lactantes de 12-23 meses, comparado con sujetos que reciben $\mathrm{SRP}+\mathrm{V}^{21}$. El estudio fase 3a NCT00226499 mostró que la ocurrencia de fiebre después de la primera dosis de SRPV podría duplicar los eventos febriles en niños que recibieron $\mathrm{SRP}+\mathrm{V}, 12,9 \%\left(10,6 ; 15,4 \mathrm{IC}_{95 \%}\right)$ vs 7,3\% (5,6; 9,3 $\left.\mathrm{IC}_{95 \%}\right)$, en tanto que la convulsión febril como evento adverso serio se presentó en 3 casos en el grupo SRPV después de la primera dosis, 0 en el SRP $+\mathrm{V}$ y 1 en el grupo SRP después de la primera dosis ${ }^{16}$.

\section{Administración simultánea con otras vacunas}

La administración de vacuna varicela monovalente es segura y con inmunogenicidad no-inferior si es administrada en forma concomitante con DTPa, DTPa-IPV, influenza (LAIV), Hib, Hib-VHB, y SRP. Lo mismo para SRPV con DTP-IPV, DTPa-IPV-VHB/Hib, DTPa+Hib/ $\mathrm{VHB}$, hepatitis $\mathrm{A}$, con la vacuna meningocócica con-
*Efectividad entendida como valor (\%) o como ausencia de hospitalizaciones o complicaciones graves. 
jugada ACWY-CRM a los 12 meses de edad o con ACWY-TT2 2 .

Por lo general, la administración simultánea de las vacunas vivas atenuadas con vacunas inactivadas produce tasas de seroconversión y de reacciones adversas similares a las observadas en la administración separada de las vacunas. La administración simultánea de las vacunas de rutina se recomienda a niños exentos de contraindicaciones específicas ${ }^{22}$.

\section{Escenarios de interés secundarios a la introducción de la vacuna}

Desplazamiento de la edad de enfermar de varicela. Existe el supuesto que la introducción de la vacuna varicela en la infancia haría disminuir la exposición de la población a VVZ, lo que produciría un desplazamiento en la edad de enfermar hacia edades mayores. La varicela a una edad mayor podría conducir a un leve aumento de la morbilidad, aunque el número total de casos debiera disminuir ${ }^{2}$.

Situación epidemiológica de herpes zoster. El año 2010, el Comité Asesor en Vacunas y Estrategias de Inmunización del Reino Unido Joint Committee on Vaccination and Immunisation (JCVI), evaluó dos estrategias de vacunación para varicela y herpes zoster, una estrategia combinada (vacunación varicela en niños y vacuna contra herpes zoster en adultos) y vacuna contra herpes zoster solamente. El JCVI declinó la estrategia combinada considerando que los modelos epidemiológicos arrojaron que, aun manteniendo coberturas de vacunación mayores a $70-80 \%$, se esperaría un aumento de incidencia de herpes zoster durante los primeros 40 a 60 años de la introducción de la vacunación, esto a raíz de que la respuesta inmune del adulto sería estimulada por la exposición a niños infectados por VVZ. La vacunación del adulto contra herpes zoster compensaría sólo en parte dicho incremento, ya que el aumento de incidencia de herpes zoster ocurriría en adultos aún jóvenes para recibir la vacuna contra herpes zoster. Además, se esperaría un aumento de casos de varicela en adultos, lo que incluye mujeres en edad fértil $\mathrm{y}$, subsecuentemente, un potencial aumento del riesgo de infección en fetos y neonatos ${ }^{23}$.

En el 2017, el Consejo Superior de Salud de Bélgica emitió la recomendación para la prevención de infecciones por varicela en niños, adolescentes y grupos de riesgo, la que no contempla la vacunación universal contra varicela. Los argumentos esgrimidos fueron que la vacuna contra varicela no puede comprometer la cobertura de la vacuna contra sarampión-rubéola-paperas y que la cobertura de vacuna varicela requiere de al menos $80 \%$ de cobertura para evitar el desplazamiento de la enfermedad de varicela hacia grupos de mayor edad, además de requerir una estrategia de catch-up en adolescentes sin antecedentes de varicela. También, considera necesario conocer la disposición de los padres hacia esta vacuna, junto con una reflexión sobre el financiamiento del programa y el tipo de vacuna y esquema vacunal más apropiados ${ }^{24}$.

Se ha discutido que la falta de convergencia entre los modelos epidemiológicos y los datos observados de herpes zoster posteriores a la introducción de vacuna contra varicela puede deberse a que los modelamientos suelen no incluir la estimulación endógena. Un estudio de modelamiento del efecto de la estimulación exógena y endógena de la inmunidad contra VVZ publicado el año 2019 mostró que un escenario de 0\% estimulación exógena y $100 \%$ estimulación endógena proyecta una caída de herpes zoster inmediata, mientras que $100 \%$ estimulación exógena y $0 \%$ estimulación endógena predice un aumento de herpes zoster durante los primeros 21 años de introducida la vacuna. Otros escenarios de estimulación exógena y endógena también muestran aumento de herpes zoster, $75-25 \%, 50-50 \%$ y $25-75 \%$, respectivamente ${ }^{25}$.

En Estados Unidos de América (E.U.A.), la vacuna contra varicela se introdujo el año 2006. Un estudio retrospectivo de incidencia de herpes zoster en niños entre 0 y 17 años entre 2003 y 2014, a partir de los registros de seguridad de vacuna de los Centros para el Control y Prevención de Enfermedades de ese país (CDC) mostró que la incidencia de herpes zoster en sujetos vacunados contra varicela fue $78 \%$ menor que en sujetos no vacunados y una reducción global de herpes zoster de $72 \%$ entre el 2003 y el $2014^{26}$. Esto indicaría que la cepa Oka presenta menor probabilidad de reactivación que VVZ salvaje ${ }^{27}$.

También en E.U.A., un estudio retrospectivo de la epidemiología de herpes zoster en el período 1991-2016 mostró que la incidencia anual de herpes zoster aumentó en todas las edades adultas entre 1,2 y $6,2 \%$. En personas de 65 y más años, el aumento de herpes zoster en los años 1991-1995 -tiempo pre-vacuna- fue 12,4\%, que disminuyó a 3,6\% en el período 1996-2006 -una dosis vacuna varicela-, y luego a 1,7\% en los años 2007-2016 -dos dosis de vacuna varicela-. En el grupo 0 a 17 años, la incidencia de herpes zoster aumentó a una tasa anual de $10,5 \%$ entre $1991-1995$ y a $3,5 \%$ en el período 1996 2006, lo que fue seguido de una caída a una tasa anual de $-12 \%$ en el período $2007-2016\left(-14 \% ; 10 \% \mathrm{IC}_{95 \%}\right)^{28}$.

\section{Vacunas registradas en Chile}

- Varilrix ${ }^{\circledR}$ : no menos de 2.000 ufp, con licencia de uso en niños de 9 meses hasta 12 años de edad inclusive: 1 dosis. Se puede administrar una dosis adicional de Varilrix $^{\circledR}$ en niños entre los 9 meses y 12 años de edad después de una primera dosis de Varilrix ${ }^{\circledR} \mathrm{u}$ otra vacuna que contenga virus varicela. Es preferible administrar la segunda dosis al menos 6 semanas después de la primera dosis, pero bajo ninguna circunstancia deberá administrarse en un plazo menor a 4 semanas. Niños 
de 13 años y más: 2 dosis con un intervalo de 6 a 10 semanas. Administrar la segunda dosis al menos 6 semanas después de la primera dosis, pero en ninguna circunstancia en un plazo de menos de 4 semanas. El esquema de dos dosis está aprobado a partir de un año de edad. Producida por GlaxoSmithKline ${ }^{29}$.

- Varivax $^{\circledR}: 1.350$ ufp, con licencia de uso en personas a partir de 12 meses de edad. Se administra como una dosis de aproximadamente $0,5 \mathrm{~mL}$ por inyección subcutánea en la región superior externa del brazo (región deltoidea) o en la región anterolateral del muslo. No se debe administrar este producto por vía intravenosa o intramuscular. Para niños de 12 meses a 12 años de edad: dos dosis de la vacuna, que deben ser administradas con un intervalo mínimo de 3 meses entre las dosis. Adolescentes (desde los 13 años de edad) y adultos: dos dosis de vacuna, que deben ser administradas con un intervalo mínimo de 4 semanas entre sí. Producida por Merck Sharp \& Dohme ${ }^{30}$.

\section{Posturas de la vacunación contra varicela}

\section{Organización Mundial de la Salud (OMS)}

La OMS recomienda que los países donde la varicela constituye una carga de salud pública importante debieran considerar la introducción de la vacuna contra varicela en el programa de vacunación de rutina. Para ello, los recursos deben ser suficientes para asegurar, alcanzar y mantener coberturas iguales o mayores a $80 \%$. Una cobertura menor a la señalada puede conducir al desplazamiento etario de la enfermedad hacia grupos mayores, lo que podría resultar en un aumento de la morbilidad y mortalidad por varicela a pesar de la disminución del número total de casos. La toma de decisión en torno a la vacuna contra varicela debe considerar el posible impacto en herpes zoster ${ }^{2}$.

Los países que decidan introducir la vacuna contra varicela a su Programa Nacional de Inmunizaciones deben administrar la primera dosis en el rango de los 12 a 18 meses de edad. Dependiendo del propósito de la vacunación será el número de dosis recomendado. Una dosis es suficiente para reducir mortalidad y morbilidad grave por varicela, pero no para limitar la circulación viral ni brotes. Dos dosis tienen mayor efectividad y debieran recomendarse a países cuyo objetivo programático sea, además de reducir mortalidad y morbilidad grave, reducir el número de casos y brotes. El intervalo mínimo entre las dos dosis varía entre cuatro semanas y tres meses, según indicación del fabricante ${ }^{2}$.

Los países pueden utilizar, tanto vacuna monovalente como combinada (SRPV), tomando en consideración sus perfiles de seguridad y efectividad, lo que incluye el riesgo de convulsión febril después de la primera dosis de
SRPV (12-18 meses), pero no después de la segunda dosis.

Países con edad elevada de adquisición de la infección, 15 o más años de edad -que indica una gran proporción de susceptibles en la población- debieran considerar estrategias alternativas como la vacunación de adolescentes $\mathrm{y}$ adultos sin antecedentes de inmunidad a varicela. Esta estrategia requiere un esquema de dos dosis.

\section{Sociedad Latinoamericana de Infectología Pediátrica (SLIPE)}

La SLIPE recomienda la incorporación de la vacuna de la varicela en todos los países de América Latina y el Caribe a los programas nacionales de inmunización con un esquema de dos dosis, la primera a partir de los 12-18 meses de edad y la segunda dosis entre los 4 y 6 años de edad ${ }^{31}$.

\section{Esquemas de vacunación}

\section{Europa}

En Europa ${ }^{32}$, la vacunación contra varicela es:

- Obligatoria en Italia (13-15 meses y 6 años) y en Letonia (12-15 meses y 6-7 años);

- Recomendada y financiada por el Estado en Austria (12-23 meses; catch up 9-17 años), Bélgica en grupos de riesgo (contactos de personas inmunocomprometidas, trabajadores de la salud, adolescentes y adultos sin antecedentes de varicela contra prueba serológica; dos dosis), Chipre (13-18 meses, 4-6 años) y República Checa (grupos de riesgo).

- Recomendada sin financiamiento por parte del Estado, en Finlandia, Alemania, Grecia, Luxemburgo, Polonia, España y Reino Unido.

\section{Región de Las Américas}

En la Región de las Américas, la vacuna contra varicela ha sido introducida ${ }^{9}$ en los siguientes países en esquemas:

Canadá: 12 meses; 18 meses o 4-5 años

Estados Unidos: 12-15 meses; 4-6 años

Bermuda: 24 meses

Bahamas: 12 meses; 4-5 años

Antigua: 24 meses

Barbados: 12 meses

Puerto Rico: 12-15 meses; 4-6 años

Islas Caimán: 12 meses; 3-6 años

México: grupos de riesgo

Costa Rica: 15 meses

Panamá: 15 meses; 4 años

Colombia: 12 meses; 5 años

Ecuador: 15 meses

Perú: 12 meses 
Brasil: 15 meses; 4 años

Paraguay: 15 meses

Uruguay: 12 meses; 5 años

Argentina: 15 meses

\section{Chile: vacunas contra varicela en grupos especiales ${ }^{33,34}$}

\section{Inmunocomprometidos: mayores de 1 año, sin antecedentes de varicela}

\section{Niños con inmunodeficiencia congénita}

- Déficit de células B: en agamaglobulinemia/inmunodeficiencia común variable, considerar uso; en déficit $\operatorname{IgA}$, déficit de subclase $\operatorname{IgG}$, vacunación de rutina.

- Déficit de células T y mixtas: vacuna varicela contraindicada.

- Trastorno de neutrófilos y fagocitosis: dos dosis espaciadas por tres meses.

- Alteración del complemento: dos dosis espaciadas por tres meses.

\section{Niños con infección por VIH}

Vacunar contra varicela en etapas clínicas asintomático $(\mathrm{N})$, síntomas leves $(\mathrm{A})$ y síntomas moderados (B) -excepto neumonitis intersticial linfoide (NIL) y etapa inmunológica 1 ( $\sin$ inmunosupresión) y en etapa 2 (inmunosupresión moderada) siempre que no haya otros factores de riesgo, como carga viral elevada.

\section{Adultos y adolescentes con infección por VIH}

- Una dosis de vacuna contra herpes zoster a partir de los 50 años si recuento LT CD4 $\geq 500$ céls $/ \mathrm{mm}^{3}$.

- Dos dosis de vacuna contra varicela separadas por tres meses en individuos sin antecedentes de varicela si recuento LT CD4 $\geq 500$ céls $/ \mathrm{mm}^{3}$.

\section{Niños con cáncer hematológico o tumores sólidos en seguimiento, a seis meses de terminada la quimioterapia}

Administrar vacuna varicela a niños que no han tenido varicela a seis meses de terminada la quimioterapia, en esquema de dos dosis separadas por tres meses. La respuesta inmune a la vacuna anti-varicela alcanza a $98 \%$ de los vacunados con dos dosis de vacuna administrada en etapa de remisión del cáncer, sin observarse efectos secundarios mayores.

\section{Trasplantes}

- Candidatos y receptores: vacuna anti-varicela al menos un mes antes del trasplante.

- Esquema de vacunación acelerado para niños en período pre-trasplante: dos dosis de vacuna varicela separadas por un mes. Edad mínima de administración es 12 meses.
- Vacuna varicela a los 12 meses posterior al trasplante de no haberla recibido antes (según estadio de inmunosupresión).

- Receptores de trasplante de precursores hematopoyéticos: dos dosis de vacuna anti-varicela separada por tres meses a los 24 meses del trasplante.

\section{Portadores de enfermedades crónicas}

Nefropatía crónica/síndrome nefrótico: dos dosis de vacuna anti-varicela separadas por tres meses.

Diabetes mellitus: dos dosis.

Niños menores de 2 años que viven con sus madres privadas de libertad

A partir de los 12 meses de edad.

\section{Inmunocompetentes}

Pre exposición: mayores de 1 año, sin antecedentes de varicela

- Personal de salud en unidades de neonatología, oncología e intensivo.

- Casas de acogida de pacientes inmunocomprometidos.

- Profesores y parvularios de escuelas hospitalarias que se encuentren en contacto con pacientes inmunocomprometidos.

- Contacto domiciliario de paciente oncológico.

- Enfermedades crónicas:

Pulmonares: fibrosis quística, displasia broncopulmonar y daño pulmonar crónico.

Cardiopatías congénitas complejas.

Afección cutánea diseminada grave.

Nefropatía crónica.

Síndrome nefrótico.

Diabetes mellitus tipo I.

- Uso crónico de ácido acetilsalicílico.

\section{Post exposición (primeras 96 h del contacto):} mayores de 1 año, sin antecedentes de varicela

- Niños hospitalizados con enfermedades intercurrentes leves.

- Personal de salud.

\section{EI CAVEI recomienda:}

Incorporar vacuna varicela monovalente cepa Oka en esquema de dos dosis. La primera dosis, entre los 12 y 18 meses. La segunda dosis, antes del ingreso a la educación prescolar.

Comité Asesor en Vacunas e Inmunizaciones de Chile Santiago, 31 de julio de 2019 


\section{Referencias bibliográficas}

1.- Pírez M. Varicella and Varicella Vaccines. In: Vaccinology Book [Internet]. SABIN; 2018. Available from: https://www.sabin.org/ programs/training-education/vaccinology-book

2.- World Health Organization. Varicella vaccines position paper [Internet]. 2014. Available from: https://www.who.int/immunization/policy/ position_papers/varicella/en/

3.- Seward J, Jumaan A. VSV: persistence in the population. In: Cambridge University Press, editor. Human Herpesviruses: Biology, Therapy, and Immunoprophylaxis. 2007.

4.- Varela F H, Pinto L A, Socotta M C. Global impact of varicella vaccination programs. Hum Vaccin Immunother. 2018; 3: 645-57. doi: 10.1080/21645515.2018.1546525.

5.- Hirose M, Gilio A, Ferronato A, Ragazzi S. The impact of varicella vaccination on varicella-related hospitalization rates: global data review. Rev Paul Pediatr. 2016; 34(3): 359-66. PMID: 26965075.

6.- Baxter R, Tran T, Ray P, Lewis E, Fireman B, Black S. Impact of vaccination on the epidemiology of varicella: 1995-2009. Pediatrics. 2014; 134: 24-30. doi: 10.1542/ peds.2013-4251.

7.- Ministerio de Salud de Chile. Vigilancia de Varicela [Internet]. Available from: http://epi. minsal.cl/varicela-vigilancia/

8.- $\quad$ Torres J, De la Maza V, Castro M, Conca $\mathrm{N}$, Izquierdo G, Suau T. Caracterización clínica y económica de hospitalizacion por varicela en niños de la Región Metropolitana y Region de Valparaíso, Chile. Value Heal 22nd Int Abstr B. 2017; 20: A929. https:// www.valueinhealthjournal.com/article/S10983015(17)33235-7/pdf

9.- Falleiros L, Pirez M, Ávila M, Cashat M, Parellada C, Wolfson L. Burden of varicella in Latin America and the Caribbean: findings from a systematic literature review. BMC Public Health 2019; 19: 528. https://doi.org/10.1186/ s12889-019-6795-0.

10.- Abarca K, Hirsch T, Potin M, Perret C, Zamorano J, González C, et al. Complicaciones en niños con varicela en cuatro hospitales de Santiago-Chile: Espectro clínico y estimación de costos directos. Rev Med Chile 2001; 129(4): 397-404. PMID: 11413992.

11.- Giglio N, Monsanto H, Rampakakis E, Yang $\mathrm{H}$, Kuter B, Wolfson L. Economic burden of varicella in children 1-12 years of age in Argentina, 2009-2014. J Med Econ 2018; 21 (4): 416-24. https://doi.org/10.1080/13696998.2 018.1431919 .

12.- Miranda-Choque E, Candela-Herrera J, DíazPera J, Farfán-Ramos S, E M-J, Escalante-
Santivañez I. Varicela complicada en un hospital pediátrico de referencia, Perú, 20012011. Rev Peru Med Exp Salud Publica. 2013; 30 (1): 45. http://www.scielo.org.pe/pdf/rins/ v30n1/a09v30n1.pdf.

13.- dos Anjos K, Ferreira M, Arruda M, Ramos K, Magalhães A. Epidemiological characterization of varicella cases in patients of a university hospital located in Recife. Rev Bras Epidemiol. 2009; 12(4): 1-10. https://doi.org/10.1590/ S1415-790X2009000400002.

14.- Pérez-Rubio A, Castrodeza J, Gil M, Luquero F, Ortiz R. Impacto social y económico de la vacunación frente a la varicela a los 15 meses de edad en Castilla y León en 2004. Rev Esp Salud Pública. 2008; 82: 101-9. https://www. redalyc.org/pdf/170/17082109.pdf.

15.- Ozaki T, Asano Y. Development of varicella vaccine in Japan and future prospects. Vaccine. 2016; 34 (29): 3427-33. doi: 10.1016/j. vaccine.2016.04.059.

16.- Prymula R, Bergsaker M, Esposito S, Gothefors L, Man S, Snegova N, et al. Protection against varicella with two doses of combined measles-mumps-rubella-varicella vaccine versus one dose of monovalent varicella vaccine: a multicentre, observer-blind, randomised, controlled trial. Lancet 2014; 383: 1313-24. doi: 10.1016/S0140-6736(12)614615.

17.- Povey M, Henry O, Bergsaker M, Chlibek R, Esposito S, Flodmark C, et al. Protection against varicella with two doses of combined measles-mumps-rubella-varicella vaccine or one dose of monovalent varicella vaccine: 10-year follow-up of a phase 3 multicentre, observer-blind, randomised, controlled trial. Lancet Infect Dis 2019; doi: 10.1016/S14733099(18)30716-3.

18.- SAGE. Systematic review of available evidence on effectiveness and duration of protection of varicella vaccines [Internet]. 2014. Available from: https://www.who.int/immunization/sage/ meetings/2014/april/4_Systematic_review_on_ effectiveness_and_duration_of_protection_of_varicella_vaccines.pdf?ua $=1$.

19.- Quinn H, Gidding H, Marshall H, Booy R, Elliott E, Richmond P, et al. Varicella vaccine effectiveness over 10 years in Australia; moderate protection from 1-dose program. J Infect 2019; 78 (3): 220-5. https://doi. org/10.1016/j.jinf.2018.11.009.

20.- Leung J, Broder K, Marin M. Severe varicella in persons vaccinated with varicella vaccine (breakthrough varicella): a systematic literature review. Expert Rev Vaccines 2017; 16 (4): 391-400. doi: 10.1080/14760584.2017.1294069.

21.- World Health Organization. Global Advisory
Committee on Vaccine Safety December 2012. Wkly Epidemiol Rec [Internet]. 2013;6(88):6572. Available from: https://www.who.int/ wer/2013/wer8806.pdf?ua=1.

22.- United States Advisory Committee on Immunization Practices. Timing and Spacing of Immunobiologics [Internet]. [citado el 28 de marzo de 2019]. Available from: https://www.cdc.gov/vaccines/hcp/acip-recs/ general-recs/timing.html.

23.- Joint Committee on Vaccination and Immunisation. Statement on varicella and herpes zoster vaccines [Internet]. 2010. Available from: https://webarchive. nationalarchives.gov.uk/20120907151317/ http://www.dh.gov.uk/prod_consum_dh/ groups/dh_digitalassets/@dh/@ab/documents/ digitalasset/dh_133599.pdf.

24.- Council Supérieur de la Santé. Vaccination des enfants, des adolescents et des personnes à risque contre la varicelle [Internet]. 2017. Available from: https://www.health.belgium. be/sites/default/files/uploads/fields/fpshealth theme_file/css_avis_9212_varicelle.pdf.

25.- Sauboin C, Holl K, Bonanni P, Gershon A, Benninghoff B, Carryn S, et al. The impact of childhood varicella vaccination on the incidence of herpes zoster in the general population: modelling the effect of exogenous and endogenous varicella-zoster virus immunity boosting. BMC Infect Dis 2019; 19:126. doi: 10.1186/s12879-019-3759-z.

26.- Weinmann S, Naleway A, Koppolu P, Baxter $\mathrm{R}$, Belongia E, Hambidge $\mathrm{S}$, et al. Incidence of herpes zoster among children: 20032014. Pediatrics 2019; 144(1):e20182917. doi: 10.1542/peds.2018-2917.

27.- Gershon A. Varicella vaccine: what have you done for me lately? Pediatrics 2019; 144 (1): e20183561. doi: 10.1542/peds.2018-3561.

28.- Wolfson L, Daniels V, Altland A, Black W, Huang W, Ou W. The Impact of varicella vaccination on the incidence of varicella and herpes zoster in the United States: Updated evidence from observational databases, 19912016. Clin Infect Dis 2019; Apr 24. pii: ciz305. doi: 10.1093/cid/ciz305.

29.- Instituto de Salud Pública de Chile. Varilrix vacuna contra la varicela liofilizado más diluyente [Internet]. 1998. Available from: http://registrosanitario.ispch.gob.cl/Ficha. aspx?RegistroISP=B-142/18.

30.- Instituto de Salud Pública de Chile. Varivax vacuna de virus vivos atenuados contra varicela, liofilizado para suspensiónn inyectable, con solvente [Internet]. 2013. Available from: http://registrosanitario.ispch. gob.cl/Ficha.aspx?RegistroISP=B-2324/18.

31.- Sociedad Latinoamericana de Infectología 
Pediátrica. Documento de posición de la SLIPE sobre prevención de varicela en América Latina y el Caribe [Internet]. 2016 [cited 2019 Jul 1]. Available from: https://www.the-ahf.org/sites/ default/files/FINAL - Varicella Position Paper June 20 2016.pdf.

32.- European Centre for Disease Prevention and
Control. ECDC Vaccine Scheduler [Internet]. [citado el 12 de junio de 2019]. Available from: https://ecdc.europa.eu/en/publications-data/ ecdc-vaccine-scheduler.

33.- González C, Cofré J. Recomendaciones para la vacunación de pacientes con necesidades especiales por patologías o situaciones de riesgo [Internet]. 2018. Available from: https:// vacunas.minsal.cl/wp-content/uploads/2019/02/ MANUAL-VACUNAS-ESPECIALES.pdf 34.- Subsecretaría de Salud Pública-Ministerio de Salud de Chile. Circular B27/04

Recomendación para prevención de varicela: uso de vacuna e inmunoglobulina. 2019 